\title{
Elliptic Genera and Vertex Operator Super Algebras
}

\author{
By Hirotaka TAMANOI*) \\ Institut des Hautes Études Scientifiques, France \\ (Communicated by Heisuke HIRONAKA, M. J. A., Oct. 12, 1995)
}

\begin{abstract}
The elliptic genus for a closed Riemannian Spin manifold, when regarded as a pair of graded vector spaces, is shown to have the structure of a pair of modules over.a vertex operator super algebra of parallel sections of an LSpin bundle. Some interesting parallel sections and the corresponding vertex operators are described for various subclasses of Riemannian manifolds defined by parallel geometric structures. In particular, vertex operators corresponding to Kähler forms generate affine Lie algebras and thus the elliptic genera are their representations.
\end{abstract}

Key words: Elliptic genera; Spin representations; vertex operator super algebras; Virasoro algebras; affine Lie algebras; Kähler manifolds; modular functions.

1. Introduction. The elliptic genus of a closed Spin manifold $M^{2 N}$ is the $S^{1}$-equivariant signature of its loop space $L M$ with respect to the canonical $S^{1}$-action on $L M$ [7]. In algebraic topology, it has been studied as a modular form valued genus, or a ring map, from cobordism rings [4]. This point of view leads to the construction of so-called elliptic cohomology. The name "elliptic" comes from the fact that the logarithm series associated to this genus can be expressed in terms of an elliptic integral of a Jacobi quartic [5]. The construction of elliptic genera involves graded vector bundles arising from the Spin representation $V, W$ of the orthogonal affine Lie algebra $\hat{\mathfrak{v}}(2 N)$, each of which is a sum of two level 1 irreducible representations.

It is known that one of these Spin repre. sentations $V$ has the structure of a vertex operator super algebra (VOA) with $W$ as its module [2]. To any vector $v$ in a vertex operator super algebra $V$, there corresponds a family of infinitely many operators $\{v\}_{n}, n \in(1 / 2) Z$, acting on the algebra itself and its modules. The main structure of a vertex operator super algebra is that the totality of these operators satisfy a Jacobi identity which is a generalization of the usual Jacobi identity for Lie algebras. Our result gives

1991 Mathematics Subject Classification. Primary 17B65, 55N22, 81R10; Secondary 17A70, 17B68, $53 \mathrm{C} 55,81 \mathrm{~T} 40$.

*) Present address: University of California, Santa Cruz, U.S.A. rise to a geometric construction of various vertex operator super algebras and their modules.

First we will observe that when a closed Spin manifold $M$ has a Riemannian structure, the elliptic genus $\Phi_{\text {ell }}(M)$ has the structure of a pair of graded vector spaces. If we take the graded dimension, we get the modular function valued elliptic genus. The Spin representation $V$ of an orthogonal affine Lie algebra $\hat{\boldsymbol{o}}(2 N)$ gives rise to a graded vector bundle $\nu_{M}$ of generalized differential forms on $M$ with a covariant derivative induced from the Levi-Civita connection on $T M$. Let $\mathscr{P}_{M}=\mathscr{P}\left(\nu_{M}\right)$ be the graded vector space of parallel sections in $\nu_{M}$.

Theorem 1. For a compact Riemannian Spin manifold $M$ without boundary, the graded vector space $\mathscr{P}_{M}$ of generalized parallel differential forms has the structure of a vertex operator super algebra and the elliptic genus $\Phi_{\mathrm{ell}}(M)$ has the structure of a super-pair of modules over $\mathscr{P}_{M}$. The diagonal action of $\mathscr{P}_{M}$ on $\Phi_{\mathrm{ell}}(M)$ is effective if the Spin index $\hat{A}(M)$ of $M$ doesn't vanish.

Thus, the elliptic genus is a geometric device which produces a super-pair of modules $\Phi_{\text {ell }}(M)$ over a vertex operator super algebra $\mathscr{P}_{M}$ for each closed Riemannian Spin manifold $M^{2 N}$.

$\left\{\begin{array}{l}\text { Closed Riemannian } \\ \text { Spin manifolds }\end{array}\right\} \stackrel{\text { elliptic genus }}{\longrightarrow}$

$$
M \stackrel{\left\{\begin{array}{l}
\text { pairs of vertex operator super } \\
\text { algebras and their modules }
\end{array}\right\}}{\longrightarrow}\left(\mathscr{P}_{M}, \Phi_{\mathrm{ell}}(M)\right)
$$

The vertex operator super algebra $\mathscr{P}_{M}$ de- 
pends only on the holonomy group of the Riemannian manifold $M$ and it always contains a weight 2 vector corresponding to the Riemannian metric tensor on $M^{2 N}$. The corresponding vertex operator generates a Virasoro algebra of rank $N$. So, the elliptic genus of a Riemannian manifold is always a representation of a Virasoro algebra. Since the total exterior bundle $\wedge^{*} T_{C}^{*} M$ is a subbundle of $\nu_{M}$, any parallel differential forms on $M$ give rise to generalized parallel differential forms, and then to vertex operators acting on the elliptic genus. On a Kähler manifold, the Kähler form is parallel. In particular, on a hyperkähler manifold, there are three Kähler forms $\kappa_{I}, \kappa_{J}, \kappa_{K}$ corresponding to parallel integrable almost complex structures $I, J, K$ satisfying quaternionic relations. Consequences of Theorem 1 for this case is described in Theorem 9.

We will describe similar results for other geometric parallel sections and for various subclasses of Riemannian manifolds including quaternion-Kähler manifolds.

Details of the results in this announcement and more can be found in [6].

2. Elliptic genera. The fixed point index formula for the $S^{1}$-equivariant signature applied formally to loop spaces gives rise to elliptic genera. Let $T_{C}=T^{*} M \otimes C$ be the complexification of the cotangent bundle of a closed Riemannian Spin manifold. For $0 \leq k \in \boldsymbol{Z}$, let $\wedge^{k}\left(T_{\boldsymbol{C}}\right)$, $S^{k}\left(T_{C}\right)$ be the $k$-th exterior power and $k$-th symmetric power bundle of $T_{\boldsymbol{C}}$. Let $\Lambda_{t}\left(T_{\boldsymbol{C}}\right)=$ $\sum_{k \geq 0} \wedge^{k}\left(T_{\boldsymbol{C}}\right) t^{k}, S_{t}\left(T_{\boldsymbol{C}}\right)=\sum_{k \geq 0} S^{k}\left(T_{\boldsymbol{C}}\right) t^{k}$ be formal series in $t$ with bundle coefficients. We define bundles $Q_{l}, R_{l}$ for $l \in(1 / 2) Z_{+}$by

$$
\begin{aligned}
& \sum_{0 \leq l \in \frac{1}{2} \boldsymbol{Z}} Q_{l} q^{l}=\bigotimes S_{0 \leq m \in \boldsymbol{Z}}\left(T_{\boldsymbol{C}}\right) \otimes \otimes \Lambda_{q^{m-\frac{1}{2}}}\left(T_{\boldsymbol{C}}\right), \\
& \sum_{0 \leq l \in \boldsymbol{Z}} Q_{l} q^{l}=\underset{1 \leq m \in \boldsymbol{Z}}{\otimes S_{q^{m}}}\left(T_{\boldsymbol{C}}\right) \otimes \Delta \otimes \otimes \Lambda_{1 \leq m \in \boldsymbol{Z}}\left(T_{\boldsymbol{C}}\right)
\end{aligned}
$$

where $\Delta=\Delta^{+} \oplus \Delta^{-}$is the complex $2^{N}$-dimensional total Spin representation which is a sum of half Spin representations for $\operatorname{Spin}(2 N)$. The bundles $\Delta^{ \pm} \otimes Q_{l}$ make sense only for Spin manifolds. Here, $q$ is a formal variable which originates in the $S^{1}$-character in the $S^{1}$-equivariant signature on the loop space $L M$ [7]. We consider Dirac operators twisted by the above bundles:

$$
d_{Q_{l}}: \Gamma\left(\Delta^{+} \otimes Q_{l}\right) \rightarrow \Gamma\left(\Delta^{-} \otimes Q_{l}\right)
$$

Similarly for $d_{R_{l}}$ using $R_{l}$ instead of $Q_{l}$. Since the twisted Dirac operator $d_{*}$ is elliptic, the kernel space $\left(\operatorname{Ker} d_{*}\right)$ and the cokernel space (Coker $d_{*}$ ) are finite dimensional for $*=Q_{l}, R_{l}, l \in$ $(1 / 2) \boldsymbol{Z}$. We remark that the twisted Dirac operators are formally self-adjoint.

Definition 2. For a compact Riemannian Spin manifold $M^{2 N}$, the elliptic genus $\Phi_{\text {ell }}^{*}(M)$ for $*=Q, R$ is a super-pair of graded vector spaces defined by

$$
\Phi_{\mathrm{ell}}^{Q}(M)=\left[\underset{0 \leq l \in \frac{1}{2} \boldsymbol{Z}}{\bigoplus}\left(\operatorname{Ker} d_{Q_{l}}\right) q^{l-\frac{N}{8}} ;\right.
$$

$$
\begin{aligned}
& \left.\underset{0 \leq l \in \frac{1}{2} Z}{\bigoplus}\left(\text { Coker } d_{Q_{l}}\right) q^{l-\frac{N}{8}}\right],
\end{aligned}
$$

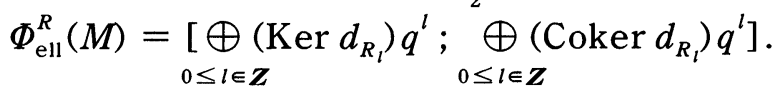

The graded super-dimension of $\Phi_{\mathrm{ell}}^{Q}(M)$, $\varphi_{\mathrm{ell}}^{Q}(M)=\sum_{0 \leq l \in \frac{1}{2} Z} \operatorname{Index}\left(d_{Q_{l}}\right) q^{l-\frac{N}{8}}$ for a Spin manifold $M$, has its value in the ring $\boldsymbol{Z}\left[q^{-\frac{1}{4}}\right]$ $\left[\left[q^{\frac{1}{2}}\right]\right]$, and the coefficients are linear combinations of Pontrjagin numbers in view of index theory. So, it is a Spin cobordism invariant. Similarly, the graded super-dimension $\varphi_{\mathrm{ell}}^{R}$ of $\Phi_{\mathrm{ell}}^{R}(M)$ has its value in $\boldsymbol{Z}[[q]]$ and it is an oriented cobordism invariant. However, our elliptic genera $\Phi_{\text {ell }}^{*}(M)$ are not cobordism invariants. The (numerical) elliptic genera $\varphi_{\text {ell }}^{*}$ are multiplicative on manifolds. For a multiplicative genus, the logarithm series is of particular interest. One feature of elliptic genera is their modular invariance. When we let $q=e^{2 \pi i r}$ for $\tau \in \boldsymbol{C}$ in the upper half plane, they are modular invariant for some subgroups of $\mathrm{SL}_{2}(\boldsymbol{Z})$. Let $\Gamma_{0}(2)=\left\{\left(\begin{array}{ll}a & b \\ c & d\end{array}\right)\right.$ $\left.\in \mathrm{SL}_{2}(\boldsymbol{Z}) \mid c \equiv 0 \bmod 2\right\}$.

Theorem 3. For the elliptic genus $\varphi_{\mathrm{ell}}^{R}: \Omega_{*}^{\mathrm{SO}}$ $\rightarrow \boldsymbol{Z}[[q]]$, its logarithm $\log _{\mathrm{ell}}^{R}(X)$ is given by an elliptic integral of the form

$$
\log _{\mathrm{ell}}^{R}(X)=\int_{0}^{X} \frac{d t}{\sqrt{1-2 \delta^{R} t^{2}+t^{4}}}, \text { where }
$$

$\delta^{R}(q)=$

$$
\frac{1}{2}\left\{\prod_{1 \leq l \in Z}\left(\frac{1+q^{l-\frac{1}{2}}}{1-q^{l-\frac{1}{2}}}\right)^{4}+\prod_{1 \leq l \in Z}\left(\frac{1-q^{l-\frac{1}{2}}}{1+q^{l-\frac{1}{2}}}\right)^{4}\right\} .
$$

$\delta^{R}(q)$ is modular invariant for an index 2 subgroup of $\Gamma_{0}(2)$.

Similar statement can be made for $\varphi_{\text {ell }}^{Q}$ which is modular invariant for an index 2 subgroup of $\Gamma_{\theta}$. In Theorem 3, the expression of $\delta^{R}(q)$ is proved by deducing a differential equation satis- 
fied by the elliptic function which is inverse to the above elliptic integral. Here, its expression as a quotient of certain theta functions is used. The modular invariance of $\delta^{R}$ follows from the invariance of the solution (which is an elliptic function) under the change of basis for the lattices of zeroes and poles of the solution function, together with the modular properties of certain theta constants. Note that all the coefficients of $\delta^{R}$ are positive integers.

Since the coefficient of $t^{4}$ in the above elliptic integral is 1, the Landweber Exact Functor Theorem [4] applies and we get a cohomology theory whose coefficient ring is $\boldsymbol{Z}[1 / 2]\left[\delta^{R}(q)\right]$. There is no obvious grading in this ring which corresponds to the dimension of manifolds, for $\delta^{R}$ has weight 0 . But in view of [3] in which elliptic genera of manifolds of different dimension are compared, the manifold dimension may not have significant meaning in the cohomological setting.

3. Vertex operator super algebras and their invariant subalgebras. Let $\left(E^{2 N},\langle\rangle,\right)$ be a Euclidean vector space. Let $A=A^{+} \oplus A^{-}=E$ $\otimes \boldsymbol{C}$ be a decomposition of the complexification of $A$ into maximal isotropic subspaces. For $m \in$ (1/2) $\boldsymbol{Z}$, let $A(-m)$ be a copy of $A$ of weight $m$. The pairing can be extended to $A_{\boldsymbol{Z}+\frac{1}{2}}=\bigoplus_{m \in \boldsymbol{Z}}$ $A(-m-(1 / 2))$ by letting $\left\langle a(m), b(n)^{2}\right\rangle=\langle a, b\rangle$ $\delta_{m+n, 0}$ for $a, b \in A$. So, the dual of $A(m)$ is $A(-m)$. The Clifford algebra associated to $\left(A_{\boldsymbol{Z}+\frac{1}{2}},\langle\rangle,\right)$ acts on the Clifford module given by

$$
V=V_{q}=\bigoplus(V){ }_{l} q^{l}=\bigotimes \Lambda_{q^{m+\frac{1}{2}}}^{*} A(-m-1 / 2)
$$

Note that the group $\operatorname{Spin}(2 N)$ acts on $V_{q}$ preserving the weight via the action of $\mathrm{SO}(2 N)$. It is known that $V_{q}$ has a structure of a vertex operator super algebra and has $W_{q}=\left(\wedge^{*} A^{-}\right) \otimes$ $\bigotimes_{1 \leq m \in \boldsymbol{Z}} \Lambda_{q^{m}} A(-m)$ as its $\left(\boldsymbol{Z}_{2}\right.$-twisted) module [2].

The main structure of the vertex operator super algebra $(V, Y(, \zeta), 1, \omega)$ consists of a graded vector space $V=\bigoplus_{0 \leq l \in \frac{1}{2} \boldsymbol{Z}}(V)_{l}$ and an injective vertex operator map $Y(, \zeta): V \rightarrow$ $\operatorname{End}(V)\left[\left[\zeta, \zeta^{-1}\right]\right]$, where $Y(v, \zeta)=\sum_{n \in Z^{\prime}}\{v\}_{n}$ $\zeta^{-n-1}$, such that $Y(1, \zeta)=$ Id for the vacuum vector $1 \in(V)_{0}$ and the vertex operator $Y(\omega, \zeta)$ $=\sum_{n \in Z} D(n) \zeta^{-n-2}$ for $\omega \in(V)_{2}$ generates a Virasoro algebra $\underline{V i r}=\bigoplus_{n \in Z} C D(n) \oplus C \operatorname{Id}_{V}$ satisfying

$[D(m), D(n)]=(n-m) D(m+n)$

$$
+\frac{m\left(m^{2}-1\right)}{12} \delta_{m,-n} c \cdot \operatorname{Id}_{V},
$$

for $m, n \in \boldsymbol{Z}$. Here, $c$ is a constant called the rank of $V$. The operator $\{v\}_{n}$ lowers the weight by $n+1-\operatorname{wt}(v)$ for any $n \in \boldsymbol{Z}$ and satisfy the following Jacobi identity. We let $|v|=2 \mathrm{wt}(v)$ $\bmod 2$

$$
\begin{aligned}
& \sum_{0 \leq i \in \boldsymbol{Z}}(-1)^{i}\left(\begin{array}{l}
r \\
i
\end{array}\right)\left(\left\{v_{1}\right\}_{m+r-i}\left\{v_{2}\right\}_{n+i}\right. \\
&\left.-(-1)^{\left|v_{1}\right|\left|v_{2}\right|+r}\left\{v_{2}\right\}_{n+r-i}\left\{v_{1}\right\}_{m+i}\right) \\
&=\sum_{0 \leq k \in \boldsymbol{Z}}\left(\begin{array}{c}
m \\
k
\end{array}\right)\left\{\left\{v_{1}\right\}_{r+k} v_{2}\right\}_{m+n-k}
\end{aligned}
$$

for any $v_{1}, v_{2} \in V$ and $m, n, r \in \boldsymbol{Z}$. The meaning of the above Jacobi identity is as follows. For $w \in V$ and $w^{*} \in V^{*}$, the graded dual, we assume that the power series of composed operators, $\left\langle Y\left(v_{1}, \zeta_{1}\right) Y\left(v_{2}, \zeta_{2}\right) w, w^{*}\right\rangle$ on $\left|\zeta_{1}\right|>\left|\zeta_{2}\right|$, $\left\langle Y\left(v_{2}, \zeta_{2}\right) Y\left(v_{1}, \zeta_{1}\right) w, w^{*}\right\rangle$ on $\left|\zeta_{2}\right|>\left|\zeta_{1}\right|$, and $\left\langle Y\left(Y\left(v_{1}, \zeta_{1}-\zeta_{2}\right) v_{2}, \zeta_{2}\right) w, w^{*}\right\rangle$ on $\left|\zeta_{1}-\zeta_{2}\right|<$ $\left|\zeta_{2}\right|$ converge to the same rational function in $\left(\zeta_{1}, \zeta_{2}\right) \in \boldsymbol{C P}^{1} \times \boldsymbol{C P}^{1}$ with possible poles along $\zeta_{1}=\zeta_{2}, \zeta_{1}, \zeta_{2}=0, \infty$. The above Jacobi identity is the consequence of the residue formula applied to this rational function for any $w \in V$ and $w^{*} \in V^{*}$. The notion of a module over a vertex operator super algebra can be defined in an obvious way. One can show that vectors in $(V)_{1 / 2}$ generate an infinite dimensional Clifford algebra, and vectors in $(V)_{1}$ generate an affine Lie algebra with an additional mild assumption on $V$.

From a given vertex operator super algebra, we can construct new algebras.

Proposition 4. Let $G \subset \operatorname{Spin}(2 N)$ be any Lie subgroup. Then the invariant subspace $V_{q}^{G}$ has the structure of a vertex operator super algebra.

The main point of the proof is that the vertex operators have an intertwining property with the action of the group $\operatorname{Spin}(2 N)$. This fact together with the Jacobi identity imply that the vertex operators $Y(v, \zeta)$ for $v \in V^{G}$ preserve $V^{G}$. The rest of the structures for a vertex operator super algebra follow by restriction from $V$.

4. Elliptic genera as modules over vertex operator super algebras. Let $\left(M^{2 N}, g\right)$ be a closed Riemannian Spin manifold. Let $T_{C}(-m)$ be a copy of the complexified cotangent bundle $T_{C}^{*} M$ having weight $m$ for $0 \leq m \in(1 / 2) Z$. Its dual vector space is denoted by $T_{C}(m)$. At each point $x \in M$, we can construct a vertex operator 
super algebra $V_{x}$ from the Euclidean vector space $\left(T_{x}^{*} M, g_{x}\right)$. These graded vector spaces form a vector bundle of vertex operator super algebras on $M$ given by

$$
\nu_{M}=\underset{0 \leq m \in Z^{q^{m+\frac{1}{2}}}}{ } T_{C}(-m-1 / 2) .
$$

$T_{C} M$ and $T_{C}^{*} M$ are identified with $T_{C}(1 / 2)$, $T_{C}(-1 / 2)$, respectively. So the total exterior bundle $\wedge^{*} T_{C}^{*} M$ is identified with $\wedge^{*} T_{C}(-1 / 2)$ $\subset \nu_{M}$. We can regard $\nu_{M}$ as the graded vector bundle of generalized differential forms. We can also consider a graded vector bundle $\mathscr{W}_{M}=\Delta \otimes$ $\otimes_{1 \leqq m \in \boldsymbol{Z}} \Lambda_{q^{m}} T_{\boldsymbol{C}}(-m)$ of $\boldsymbol{Z}_{2}$-twisted modules over the bundle of vertex operator super algebras $\nu_{M}$. The Levi-Civita connection on $M$ induces a covariant derivative $\nabla$ on the graded vector spaces of smooth sections of $\nu_{M}$ and of $\mathscr{W}_{M}$. Let $\mathscr{P}_{M}$ be the graded vector space of parallel sections in $\nu_{M}$. For a connected $M,\left(\mathscr{P}_{M}\right)_{0} \cong C \cdot 1$, where 1 is the constant function.

Theorem 5. $\mathscr{P}_{M}$ has the following properties:

(i) $\mathscr{P}_{M}$ has the struclure of a verlex operalor super algebra

(ii) For any parallel section $\sigma \in \mathscr{P}_{M}$, the associaled verlex operalor commules with covarianl derivalive, i.e. $\left[\nabla,\{\sigma\}_{n}\right]=0$ for $n$ $\in(1 / 2) \boldsymbol{Z}$.

(iii) If $G \subset \operatorname{Spin}(2 N)$ is the slruclure group of a holonomy bundle on $M, \mathscr{P}_{M}$ is canonically isomorphic to $V^{G}$ as verlex. operator super algebras.

To prove (ii) above, we first note that the action of a vertex operator is a combination of exterior multiplications and dual pairings. Then, we use the fact that the covariant derivative commutes with dual pairings and acts as a derivation on exterior products. (i) is essentially a consequence of (ii), (iii) follows from a fact that a globally parallel section in a vector bundle is completely determined by its restriction to a fiber over any point $x \in M$.

5. Infinite dimensional symmetries in elliptic genera. We describe some consequences of Theorem 1 for various closed irreducible Riemannian manifolds [1]. In view of the classification theory of the holonomy groups, we consider those Riemannian manifolds $M^{2 N}$ whose holonomy groups are contained in $\mathrm{U}(N), \mathrm{SU}(N)$, $\operatorname{Sp}\left(N^{\prime}\right) \operatorname{Sp}(1)$, and $\operatorname{Sp}\left(N^{\prime}\right)$, with $N=2 N^{\prime}$. The results below are proved through the isomorph- ism $\mathscr{P}_{M} \cong V^{G}$ in Theorem 5 . In what follows, we use the notation $[v]$ to denote a vector in $\mathscr{P}_{M}$, and use $\{v\}_{*}$ to denote the corresponding vertex operators.

Riemannian manifolds. The Riemannian metric $g: T M \otimes T M \rightarrow \boldsymbol{R}$ is parallel on $M$. A generalized Riemannian metric tensor $\hat{g}: T_{C}(3 / 2)$ $\otimes T_{\boldsymbol{C}}(1 / 2) \rightarrow \boldsymbol{C}$ defined by $\hat{g}\left(v_{1}(3 / 2), v_{2}(1 / 2)\right)$ $=g\left(v_{1}, v_{2}\right)$ for $v_{1}, v_{2} \in T_{C} M$ is a weight 2 parallel section in $\Gamma\left(T_{C}(-3 / 2) \otimes T_{C}(-1 / 2)\right)$. So $[\hat{g}] \in\left(\mathscr{P}_{M}\right)_{2}$.

Theorem 6. On a closed Riemannian Spin manifold $\left(M^{2 N}, g\right)$, the verlex operator $Y(-[\hat{g}] / 2, \zeta)$ $=\sum_{m \in Z} D(m) \zeta^{-m-1}$ for the generalized Riemannian melric tensor $\hat{g} \in\left(\mathscr{P}_{M}\right)_{2}$ generales a Virasoro algebra of rank $N$. Hence, the elliptic genus $\Phi_{\mathrm{ell}}^{*}\left(M^{2 N}\right)$ is a super-pair of rank $N$ represenlalions of the Virasoro algebra.

Kähleran manifolds. A Riemannian manifold $\left(M^{2 N}, g\right)$ with an isometric almost complex structure $I$ is called Kählerian if $I$ is parallel. In this case, $I$ is integrable and $M$ is actually a complex manifold and the holonomy group of $M$ is contained in $\mathrm{U}(N)$. The Kähler form $\kappa \in$ $\wedge^{2} T^{*} M$ defined by $\kappa(X, Y)=\kappa_{I}(X, Y)=$ $g(I(X), Y)$ for $X, Y \in T M$ is parallel because $g$ and $I$ are parallel. Through the embedding $\wedge^{*} T M \hookrightarrow \underline{V}$, we obtain $[\kappa] \in\left(\mathscr{P}_{M}\right)_{1}$ and $\left[\kappa^{2}\right] \in$ $\left(\mathscr{P}_{M}\right)_{2}$. In $\left(\mathscr{P}_{M}\right)_{2}$, we consider vectors $[\theta]=$ $\left(\left[\kappa^{2}\right]-[\hat{g}]\right) /(2 N)$ and $[\lambda]=-\left(\left[\kappa^{2}\right]+(N-\right.$ 1) $[\hat{g}]) /(2 N)$. Note that $[\theta]+[\lambda]=-[\hat{g}] / 2$ generates the Virasoro algebra above.

Theorem 7. Lel $M^{2 N}$ be a closed Kählerian Spin manifold. The vertex operator super algebra $\mathscr{P}_{M}$ contains the following Lie algebras:

(i) (Heisenberg algebra) The vertex operator $Y([\kappa], \zeta)=\sum_{n \in Z} K(n) \zeta^{-n-1}$ generates $a$ Heisenberg algebra $\mathfrak{h}=\bigotimes_{m \neq 0} \boldsymbol{C K}(m) \oplus$ $C \mathrm{Id}_{V}$ wilh commutation relations $[K(m)$, $K(n)]=n N \delta_{m+n, 0} \mathrm{ld}_{V}$, for $m, n \in \boldsymbol{Z}$.

(ii) (Unitary Virasoro algebra) The vertex operators $Y([\theta], \zeta)$ and $Y([\lambda], \zeta)$ generate two commuting Virasoro algebras of rank 1 , $N-1$, respectively.

The elliptic genus $\Phi_{\mathrm{ell}}^{*}(M)$ is a super-pair of representations of the above algebras.

A Kähler manifold $M^{2 N}$ whose holonomy group is contained in $\mathrm{SU}(N)$ has the vanishing Ricci tensor. So it is automatically a Spin manifold and Theorem 7 applies. For these manifolds, 
the complex volume forms $\xi \in \Gamma\left(\wedge^{N} T_{C}^{(1,0)} M\right)$ and their conjugates are also parallel and generate interesting vertex operators.

Quaternion-Kähler manifolds. A Riemannian manifold $\left(M^{4 N^{\prime}}, g\right), N=2 N^{\prime}$, is called quaternionKähler if there exists a real 3-dimensional parallel subbundle $L \subset \operatorname{End}(T M)$ which is locally spanned by isometric almost complex structures $I, J, K$ satisfying the quaternion relations. The holonomy group of $M$ is contained in $\mathrm{Sp}\left(N^{\prime}\right)$. $\mathrm{Sp}(1)$. Quaternion-Kähler manifolds are not necessarily Kähler manifolds. Corresponding to locally defined almost complex structures $I, J$, $K$ which may not be parallel, we have locally defined 2 -forms $\kappa_{I}, \kappa_{J}, \kappa_{K}$ which may not be parallel nor closed. However, the sum of squares $\kappa_{Q-K}$ $=\kappa_{I}^{2}+\kappa_{J}^{2}+\kappa_{K}^{2}$ is a globally defined parallel closed differential 4 -form on $\boldsymbol{M}$. We have $\left[\kappa_{Q-K}\right],[\hat{g}] \in\left(\mathscr{P}_{M}\right)_{2}$. We let $[\sigma]=\left(\left[\kappa_{Q-K}\right]-3\right.$ $[\hat{g}]) / 2(N+4)$ and $[\tau]=-\left(\left[\kappa_{Q-K}\right]+(N+1)\right.$ $[\hat{g}]) / 2(N+4)$. Again note that $[\sigma]+[\tau]=$ - $[\hat{g}] / 2$ generates the rank $N$ Virasoro algebra.

Theorem 8. Lel $M^{4 N^{\prime}}$ be a closed qualernionKähler Spin manifold. The elliplic genus $\Phi_{\mathrm{ell}}^{*}(M)$ is a super-pair of representalions of a symplectic Virasoro algebra which is a direcl sum of lwo com. muling Virasoro algebras of rank $3 N /(N+4)$, $N(N+1) /(N+4)$, respectively, generated by vertex operators $Y([\sigma], \zeta)$ and $Y([\tau], \zeta)$.

Hyperkähler manifolds. A hyperkähler manifold $M^{4 N^{\prime}}$ possesses three isometric parallel almost complex structures $I, J, K$ satisfying the quaternion relations. The holonomy group of $M$ is contained in $\mathrm{Sp}\left(N^{\prime}\right)$. The corresponding Kähler forms $\kappa_{I}, \kappa_{J}, \kappa_{K}$ are parallel and closed, so is $\kappa_{Q-K}=\kappa_{I}^{2}+\kappa_{J}^{2}+\kappa_{K}^{2}$. For any integrable almost complex structure $\mathscr{T}=a I+b J+c K$ with $a^{2}+b^{2}+c^{2}=1, a, b, c \in \mathbf{R}$, the corresponding Kähler form is given by $\kappa_{\mathscr{T}}=a \kappa_{I}+b \kappa_{J}$ $+c \kappa_{K}$. We let $N=2 N^{\prime}$. Thus, we have $\left[\kappa_{I}\right]$, $\left[\kappa_{J}\right],\left[\kappa_{K}\right],\left[\kappa_{\mathscr{T}}\right] \in\left(\mathscr{P}_{M}\right)_{1}$ and $\left[\kappa_{Q-K}\right],\left[\kappa_{\mathscr{T}}^{2}\right],[\hat{g}]$ $\in\left(\mathscr{P}_{M}\right)_{2}$.

Theorem 9. Let $M^{4 N^{\prime}}$ be a connected closed hyperkähler manifold. The vertex operator super algebra $\mathscr{P}_{M}$ contains the following subalgebras:

(1) (Affine Lie algebra $A_{1}^{(1)}$ ) The vertex oper. ators corresponding to Kähler forms $\left[\kappa_{I}\right]$, $\left[\kappa_{J}\right],\left[\kappa_{K}\right] \in\left(\mathscr{P}_{M}\right)_{1}$ generate a level $N^{\prime}$ affine Lie algebra $A_{1}^{(1)}$.
(2) (Unitary Virasoro algebra) For any parallel almosl complex slruclure $\mathscr{T}=a I+b J$ $+c K$ as above, the subspace $\boldsymbol{C}\left[\kappa_{\mathscr{T}}^{2}\right] \oplus$ $\boldsymbol{C}[\hat{g}] \subset\left(\mathscr{P}_{M}\right)_{2}$ generales a unilary Virasoro algebra of rank $(1, N-1)$.

(3) (Symplectic Virasoro algebra) The subspace $\boldsymbol{C}\left[\kappa_{Q-K}\right] \oplus \boldsymbol{C}[\hat{g}] \subset\left(\mathscr{P}_{M}\right)_{2}$ generates a symplectic Virasoro algebra of rank ( $3 N /$ $(N+4), N(N+1) /(N+4))$.

(4) For any veclor $[\vartheta] \in S^{2}\left(\boldsymbol{C}\left[\kappa_{I}\right] \oplus \boldsymbol{C}\left[\kappa_{J}\right] \oplus\right.$ $\left.\left[\kappa_{K}\right]\right) \subset\left(\mathscr{P}_{M}\right)_{2}$, the corresponding verlex operalors $\{\vartheta\}_{*}$ salisfy commulalion relalions of the form

$$
\begin{aligned}
& {\left[\{\vartheta\}_{m+1},\{\vartheta\}_{n+1}\right]=\left(\frac{m-n}{2}\right)\left\{\{\vartheta\}_{1}[\vartheta]\right\}_{m+n+1}} \\
& \quad+\left(\begin{array}{c}
m+1 \\
3
\end{array}\right)\left(\{\vartheta\}_{3}[\vartheta], 1\right) \delta_{m,-n} \cdot \operatorname{Id}_{V},
\end{aligned}
$$

where $\{\vartheta\}_{1}[\vartheta] \in S^{2}\left(\boldsymbol{C}\left[\kappa_{I}\right] \oplus \boldsymbol{C}\left[\kappa_{J}\right] \oplus\left[\kappa_{K}\right]\right) \oplus$ $\boldsymbol{C}[\hat{g}] \subset\left(\mathscr{P}_{M}\right)_{2}$ and the pairing (, ) on $\left(\mathscr{P}_{M}\right)_{0}=$ $C \cdot 1$ is such that $(1,1)=1$.

The elliplic genus $\Phi_{\mathrm{ell}}^{*}(M)$ is a super-pair of represenlations of the above algebras.

The above results suggests the study of the vertex operators generated by generalized Kähler forms and generalized Riemannian tensors.

\section{References}

[1] A. L. Besse: Einstein Manifolds. Ergebnisse der Math., 3 Folge, Band 10, Springer-Verlag, New York (1987)

[2] A. J. Feingold, I. B. Frenkel, and, J. F. X. Ries: Spinor Construction of Vertex Operator Algebras. Triality and $E_{8}^{(1)}$. Contemp. Math., vol. 121, Amer. Math. Soc., Providence, R. I. (1991).

[ 3 ] F. Hirzebruch and P. Slodowy: Elliptic genera, involutions and homogeneous Spin manifolds. Geom. Dedicata, 35, 309-343 (1990).

[4] P. S. Landweber: Elliptic cohomology and modular forms. Lect. Notes in Math. (ed. P. S. Landweber). vol. 1326, Springer-Verlag, New York, pp. 55-68 (1988).

[5] S. Ochanine: Sur les genres multiplicatifs définis par des intégrales elliptiques. Topology, 26, 143-151 (1987).

[6] H. Tamanoi : Elliptic genera and vertex operator super algebras. 372pages (1994) (preprint).

[7] E. Witten: The index of the Dirac operator in loop space. Lect. Notes in Math. (ed. P. S. Landweber). vol. 1326, Springer-Verlag, New York, pp. 161-181 (1988). 